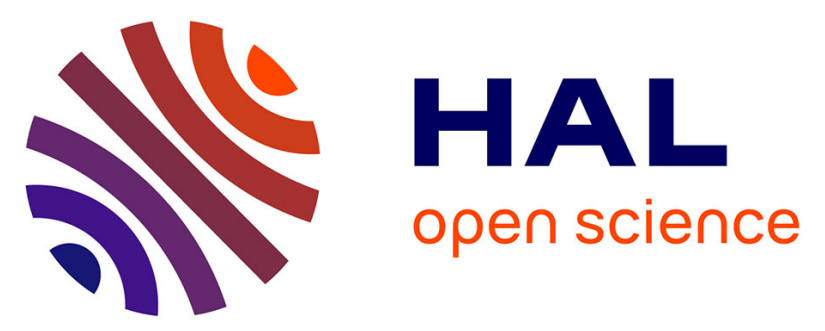

\title{
HLA-B*5801 et réactions cutanées à l'allopurinol dans la population Kinh d'Ho Chi Minh Ville (Vietnam)
}

T. Bardin, Q.D. Nguyen, T.M.K. Bui, N.H. Le, Matthieu Resche-Rigon, M.D.

Do

\section{- To cite this version:}

T. Bardin, Q.D. Nguyen, T.M.K. Bui, N.H. Le, Matthieu Resche-Rigon, et al.. HLA-B*5801 et réactions cutanées à l'allopurinol dans la population Kinh d'Ho Chi Minh Ville (Vietnam). Bulletin de l'Académie Nationale de Médecine, 2019, 203, pp.442 - 448. 10.1016/j.banm.2019.02.012 . hal03487843

\section{HAL Id: hal-03487843 \\ https://hal.science/hal-03487843}

Submitted on 20 Dec 2021

HAL is a multi-disciplinary open access archive for the deposit and dissemination of scientific research documents, whether they are published or not. The documents may come from teaching and research institutions in France or abroad, or from public or private research centers.
L'archive ouverte pluridisciplinaire HAL, est destinée au dépôt et à la diffusion de documents scientifiques de niveau recherche, publiés ou non, émanant des établissements d'enseignement et de recherche français ou étrangers, des laboratoires publics ou privés.

\section{(ㄷ)(1) $\$$}

Distributed under a Creative Commons Attribution - NonCommerciall 4.0 International 


\section{Article original}

Reçu le 27/03/2018

Accepté le 11/02/2019

HLA-B*5801 et réactions cutanées à l'allopurinol dans la population Kinh d'Ho Chi Minh Ville (Vietnam)*

HLA-B*5801 and cutaneous reactions to allopurinol in the Kinh population of Ho Chi Minh City (Vietnam)

Thomas BARDIN ${ }^{1,2,3}$, Thi Minh Kim BUI ${ }^{1}$, Nghia Hieu LE ${ }^{1}$, Quang Dinh NGUYEN ${ }^{1}$,Matthieu RESCHE-RIGON ${ }^{4,5}$, Minh Duc DO ${ }^{6}$.

Auteur correspondant :

Thomas Bardin

Service de Rhumatologie, centre Viggo Petersen, hôpital Lariboisière, 2 rue Ambroise Paré, 75010 Paris

thomas.bardin@aphp.fr

1. Centre de recherche Franco-Vietnamien sur la goutte et les maladies chroniques, centre médical Vien Gut, Ho Chi Minh City, Vietnam

2. Service de Rhumatologie, Hôpital Lariboisière Paris

3. Unité INSERM 1132 Université Paris Diderot, Paris

4. Service de biostatistiques et information, hôpital Saint Louis, Paris

5. Unité INSERM 1153 Université Paris Diderot, Paris

6. Center for Molecular Medicine, University of Medicine and Pharmacy, Ho Chi Minh City, Vietnam

* Séance du 16 avril 2019

Les auteurs ne déclarent aucun lien d'intérêt.

Mots clés : Allopurinol, goutte, toxidermies, HLA

Key index words: Allopurinol, gout, skin reaction, HLA 
Résumé

Le but de cette étude prospective était d'identifier les facteurs de risque, incluant l'HLA-B*5801, de réactions cutanées sévères ou bénignes à l'allopurinol dans l'ethnie Kinh, majoritaire au Vietnam. Dix réactions sévères (syndromes de Lyell ou de Stevens Johnson) furent identifiées dans les services de dermatologie d'Ho Chi Minh Ville (HCMV), 54 réactions bénignes dans ces même services et au centre médical Vien Gut (HCMV), spécialisé dans la prise en charge de la goutte, et 112 patients sans intolérance (pas de réaction dans les 3 mois suivant la dernière augmentation de posologie de l'allopurinol) furent recrutés au Vien Gut. Les données cliniques furent collectées de façon prospective et un typage HL* B5801 fut réalisé à l'aide du kit de détection PG5801 (Parmigene, Taiwan). L'HLA-B*85801, présent chez les 10 patients avec réaction sévère, a été trouvé très significativement ( $\mathrm{p}<0,0001$; Odds Ratio $(\mathrm{OR})$ : 171 ; écart type (ET) $95 \%: 20 ; 7889)$ associé à ces accidents graves. Les autres facteurs de risque de réactions sévères rapportés dans la littérature (absence de goutte, sexe féminin, insuffisance rénale, hypertension artérielle, dyslipidémie, absence d'augmentation progressive de la posologie), mais pas la dose d'allopurinol, ont été confirmés. Pour les réactions bénignes, il n'y avait pas d'association à l'HLA*B5801. Seules l'absence d'augmentation progressive de l'allopurinol, l'hypertension artérielle et les dyslipémies leur étaient associées.

\section{Abstract:}

The aim of this prospective study was to investigate risk factors, including HLA-B*58 01, for mild (MCAR) and severe (SCAR) cutaneous reactions in the predominant Kinh ethnicity of Vietnam. Ten SCAR patients (toxic necrotic epidermolysis (TEN) and/or Stevens-Johnson syndromes) were recruited from hospital-based dermatology departments in Ho Chi Minh City (HCMC), 54 patients with MCARs from the same departments and from the Vien Gut medical center at HCMC, that is specialized in gout care, and 112 tolerant gouty patients (no skin reaction after at least 3 months from the last increment in allopurinol dose) at the Vien Gut. Clinical data were prospectively collected and HLA-B*58 01 typing was done using PG5801 DNA detection kit (Pharmigene-Taiwan) according to manufacturer instruction. HLA*B58 01 was present in the $10 \mathrm{TEN} /$ Stevens Johnson patients and was significantly associated with SCARs (P<0.0001, OR 171; 95\% CI: 20; 7889). Other known factors such as female sex, nongout, renal failure, hypertension, dyslipemia, lack of allopurinol titration, but not allopurinol dose were associated with SCARs. The only associations with MCARs were lack of allopurinol titration, hypertension and dyslipemia. 


\section{Introduction}

Plus de 50 ans après sa mise sur le marché en France (1967), l'allopurinol reste, malgré l'enrichissement des possibilités thérapeutiques, l'hypouricémiant le plus utilisé dans le monde, du fait d'une longue expérience et de son prix très bas.

L'allopurinol est généralement bien toléré, mais peut être source d'accidents cutanés, le plus souvent bénins, mais parfois graves [1]. Ces intolérances cutanées surviennent dans les premiers mois du traitement. Les accidents graves sont classés en deux groupes principaux [2] : syndromes de Lyell ou de Stevens Johnson d'une part, dont on reconnait actuellement la similitude des lésions anatomiques et l'existence de formes mixtes, qui surviennent généralement dans les 3 premiers mois du traitement et dont la mortalité est de 10 à 30 p. cent; syndromes d'hypersensibilité, actuellement appelés syndromes DRESS (pour Drug Reaction with Eosinophilia and Systemic Symptoms) d'autre part, survenant généralement 2 à 6 semaines après l'introduction du médicament, et dont la mortalité est d'environ $10 \%$. L'allopurinol vient au premier rang des médicaments incriminés dans le déclenchement des syndromes de Lyell / Stevens Johnson [3-5], et en deuxième position parmi les médicaments responsables de DRESS [6]. Ces toxicités sont heureusement très rares [7], et le risque en semble variable selon l'ethnie [8], ce qui suggère l'existence d'une prédisposition génétique [9]. En 2005, la très forte association de ces toxidermies graves à l'HLA-B*5801 fut mise en évidence chez les chinois Han de Taiwan [10]. Il a été, depuis, montré que cette molécule HLA présente l'oxypurinol, métabolite actif de l'allopurinol, aux cellules $\mathrm{T}$ cytotoxiques, ce qui déclenche une réaction immunologique ciblant l'épiderme $[11,12]$. Cette découverte permet maintenant d'éviter, à Taiwan, les toxidermies graves liées à l'allopurinol en cherchant cet allèle à risque chez les candidats au traitement et en donnant un autre médicament hypouricémiant chez ceux qui en sont trouvés porteurs [13]. Cependant la force de l'association et la fréquence de l'allèle dans la population varient d'une ethnie à l'autre et sont par exemple, en France et au Japon, jugées insuffisantes pour justifier le génotypage HLA-B*5801 avant prescription de l'allopurinol [14]. De plus l'association de l'allèle à risque avec les toxidermies mineures reste controversée [15].

On sait peu de choses sur les réactions cutanées au Vietnam, où les gouttes graves, requérant de fortes posologies d'allopurinol, semblent, selon notre expérience [16], fréquentes. Cela nous a incités à entreprendre une étude prospective de ces réactions, incluant un génotypage HLA$\mathrm{B} * 5801$, dont nous rapportons ici les premiers résultats.

\section{Méthodes}

Tous les patients inclus étaient de l'ethnie Khin, majoritaire au Vietnam, prenaient de l'allopurinol, ont été vus à Ho Chi Minh ville (HCMV) et ont signé une feuille de consentement après information orale et écrite sur l'étude, préalablement approuvée par le comité d'éthique de l’Université de Médecine et de Pharmacie d'HCMV (Numéro d'approbation : 2017198).

Nous avons constitué 3 groupes distincts par leur tolérance au médicament : 
1. Intolérances cutanées graves, recrutées dans deux services de dermatologie d'hôpitaux de la ville. Les réactions ont été classées par les dermatologues selon les critères du groupe Regi-SCAR [17].

2. Intolérances cutanées bénignes, recrutées dans ces mêmes hôpitaux et au centre médical Vien Gut (HCMV), spécialisé dans la prise en charge de la goutte. Ces intolérances prenaient la forme d'éruptions maculo-papuleuses ou de prurits isolés, qui dans tous les cas, ont amené à l'arrêt de l'allopurinol et ont disparu après cet arrêt.

3. Bonne tolérance à l'allopurinol, définie par l'absence d'intolérance, après un délai d'au moins 3 mois depuis la dernière augmentation de posologie du médicament. Tous les patients de ce groupe ont été recrutés au centre médical Vien Gut.

Un recueil prospectif des données cliniques et biologiques a été effectué et un fichier Excel anonyme constitué, dans lequel ont été inscrits les renseignements obtenus sur le type d'accident et son évolution, les anomalies biologiques observées au cours de l'éruption, les facteurs de risque connus ou suspectés de réaction cutanée (âge, sexe, absence de diagnostic de goutte, comorbidités et facteurs de risque cardiovasculaires, prise de diurétique, antécédents d'allergie à l'allopurinol, présence d'une insuffisance rénale avant l'éruption, dose d'allopurinol lors de l'introduction du médicament et lors de l'éruption, résultats du génotypage HLA-B*5801). Les autres médicaments pris lors de l'éruption ont été aussi notés.

Le génotypage HLA*B-5801 a été effectué dans le centre de biologie moléculaire de l'Université de Médecine et de Pharmacie d'HCMV. L'ADN a été rapidement extrait par méthode automatisée et stocké à $-20^{\circ} \mathrm{C}$ jusqu'au génotypage. Le génotypage HLA*B-5801 a été obtenu en utilisant le kit de détection d'ADN PG5801 (Parmgene, Taiwan,) par réaction de polymérisation de l'ADN amorcée par une séquence spécifique de l' HLA-B*5801.

Les variables qualitatives ont été décrites par leurs effectifs et pourcentages et les variables quantitatives par leurs moyennes et écart-types. Les différences entre les trois groupes ont, en premier lieu, été testées globalement à l'aide de tests de Fisher pour les variables qualitatives et de tests de Kruskal-Wallis pour les variables quantitatives. En cas de significativité, les groupes ont été testés 2 à 2 à l'aide de tests de Fisher ou de Wilcoxon respectivement. Tous les tests étaient de formulation bilatérale au risque 0,05 . L'analyse statistique a été effectuée à l'aide du logiciel R (version 3.4.1; https://cran.r-project).

\section{Résultats}

Entre octobre 2016 et janvier 2018, 10 patients ont été hospitalisés en dermatologie pour des réactions cutanées graves à l'allopurinol. Il s'agissait d'un syndrome de Lyell, de 8 syndromes de Stevens-Jonhnson, et d'un syndrome mixte (fig.1), survenus en moyenne 12,7 jours après l'introduction de l'allopurinol. Quatre patients ont eu une élévation réversible des enzymes hépatiques, qui ont été dosées chez 6 des 10 patients. L'éruption a duré en moyenne un mois. 
Aucun patient n'est mort. Les caractéristiques de ces patients sont détaillées dans le tableau 1; les données biologiques avant mise sous allopurinol étaient souvent manquantes, 6 pattients seulement avaient eu leur créatinine mesurée avant le début du traitement.

54 patients avec réaction bénigne ont été inclus (fig. 2). Ils recevaient de l'allopurinol depuis 15 jours en moyenne. Dans tous les cas, la réaction a conduit à l'arrêt de l'allopurinol et à la guérison rapide (moyenne 15 jours, extrêmes 1 à 40 jours) de l'intolérance. 38 patients avaient une éruption maculo-papuleuse souvent prurigineuse, associée dans un cas à une desquamation palmoplantaire, et dans un autre (HLA-B*5801+) à une atteinte labiale et bucale, sans décollement. Les 16 autres patients de ce groupe n'avaient qu'un prurit simple, qui a rapidement régressé à l'arrêt de l'allopurinol. Il n'y avait pas de différence significative entre les caractéristiques des patients avec et sans éruption, qui ont donc été réunis dans le tableau 1.

112 patients ayant bien toléré l'allopurinol ont aussi été inclus (tableau 1).

Le génotypage HLAHLA-B*5801 a été positif chez les 10 patients qui avaient développé des réactions cutanées graves, chez $3(5,6 \%)$ des patients avec éruptions bénignes (dont 1 n'avait qu'un prurit), et chez $6(5,3 \%)$ de ceux qui avaient bien toléré l'allopurinol (tableau 1). L'analyse statistique a montré que la présence de l'HLA-B*5801était significativement associée aux réactions cutanées graves ( $<0,0001$; Odds Ratio $(\mathrm{OR}): 171,2$ (intervalle de confiance à 95 $\%$ (IC 95\%) 20 ; 7889), alors qu'elle ne l'était pas aux réactions bénignes $(\mathrm{p}=1)$.

Comme le montre le tableau 1, les caractéristiques non HLA significativement associées aux réactions graves étaient l'absence de goutte $(\mathrm{p}<0,0001)$, le sexe féminin $(\mathrm{p}=0006)$, un âge plus élevé $(\mathrm{p}=0,04)$, la prise de diurétque $(\mathrm{p}=0,005)$, l'existence d'une insuffisance rénale définie comme un débit de filtration glomérulaire estimé inférieur à $60 \mathrm{ml} / \mathrm{min}(\mathrm{p}=0,004)$, l'existence d'une hypertension artérielle $(\mathrm{p}=0,06)$ ou d'une hyperlipémie $(\mathrm{p}=0,02)$, l'absence d'augmentation progressive de la posologie d'allopurinol ( $\mathrm{p}<0,0001)$, et la présence d'un antécédent d'intolérance cutanée bénigne à l'allopurinol $(\mathrm{p}=0.006)$. En revanche, la posologie d'allopurinol n'était pas associée au développement des réactions cutanées graves $(p=0,51)$.

Les seuls facteurs significativement associés aux réactions cutanées bénignes étaient la présence d'une hypertension artérielle $(\mathrm{p}<0,06)$ ou d'une hyperlipémie $(\mathrm{p}<0,0001)$ et l'absence d'augmentation progressive de la posologie d'allopurinol $(\mathrm{p}<0,0001)$ (taleau 1).

\section{Discussion}

Dans notre étude prospective, nous avons, à ce stade, identifié 10 cas de réactions cutanées sévères à l'allopurinol, qui appartenaient toutes au groupe des syndromes de Lyell/Stevens Johnson. Aucun cas de DRESS n'a été encore inclus, dont l'association à l' HLA-B*5801, a été observée au Portugal [18] et en Thaïlande [19] . Les 10 syndromes de Lyell/Stevens Johnson que nous avons, à ce jour, inclus dans notre étude était tous porteurs de l'allèle HLA-B*5801. L'Odds-Ratio associée à l'allèle était élevée $(171,2$ IC95 \%: $20 ; 7889)$, et nos résultats sont voisins de ceux obtenus dans la population Han de Taiwan, de Chine continentale et de Hong Kong [10, 20, 21], en Thaïlande [19], et chez les insuffisants rénaux coréens [22]. Notre étude confirme aussi les résultats d'une étude rétrospective récemment menée à Hanoi, dans laquelle 21 des 22 réactions sévère étudiées étaient associées à l'allèle à risque [23]. Notre série est 
cependant encore courte, l'imprécision de l'OR est importante et une augmentation du nombre de cas étudiés nous semble nécessaire à l'affinement de ces données.

L'intérêt, pour la prévention des réactions cutanées graves, d'un génotypage HLA-B*5801 avant prescription de l'allopurinol, dépend de l'OR associée à l'allèle à risque, mais aussi de la fréquence de cet allèle dans la population considérée. Au Japon [24] et en Europe [25], ce génotypage n'est pas recommandé [14] car seulement la moitié environ des accidents graves ont été associés à HLA-B*5801, et aussi parce que la fréquence de l'allèle à risque (environ 1\%) dans les populations concernées est faible, ces 2 éléments rendant le génotypage peu rentable [14]. Dans notre étude, nous avons choisi pour la population contrôle des patients ayant bien toléré l'allopurinol, que nous pouvions facilement recruter au centre médical Vien Gut, parce qu'il nous paraissait intéressant de connaitre la fréquence de l'allèle à risque chez les candidats à un traitement par l'allopurinol. Cette fréquence peut être un peu différente de celle évaluée en population générale, ce qui peut influencer légèrement le calcul de l'OR, mais les différences de prévalence de l'allèle sont généralement faibles, par exemple $19 \%$ dans la population générale, et $20 \%$ chez les patients tolérants dans l'étude Taiwanaise princeps [10]. Au Vietnam, deux études ont précédemment estimé la fréquence de l'allèle HLA-B*5801dans la population générale avec des résultats quelque peu discordants. L'une [26], portant sur $170 \mathrm{Kinhs}$ rapporte une estimation $(6,5 \%)$ proche de la nôtre $(5,3 \%)$. L'autre, portant sur 75 sujets, rapporte un chiffre de $18,6 \%$ [27]. Il semble important d'augmenter les effectifs de notre groupe contrôle afin d'y estimer de manière plus fiable la prévalence de l'allèle à risque.

Nous nous sommes aussi intéressés aux facteurs de risque non HLA des réactions cutanées graves à l'allopurinol, et avons, dans l'ensemble, confirmé les résultats des études précédemment publiées. Les réactions cutanées à l'allopurinol sont survenues chez nos malades, comme dans toutes études de la littérature [1, 28], peu après l'introduction du médicament. Dans certaines études publiées, la prescription d'allopurinol en dehors de la goutte [29] et le sexe féminin [8, 29] étaient associées aux accidents cutanés graves, ce qui s'accorde avec nos résultats. Nous confirmons l'association avec l'âge [8, 29, 30], même si le degré de signification statistique est ici faible $(\mathrm{p}<004)$. Nous avons aussi trouvé une association significative de la prise de diurétiques avec les réactions d'intolérance grave, malgré la faiblesse de nos effectifs. La prise de diurétiques est un facteur de risque classique [28, 31, 32], qui a été remis récemment remis en question : ce pourraient être les maladies cardiovasculaires justifiant un traitement diurétique, et non le traitement lui-même qui soient en cause [29, 30]. Nous avons noté une association avec l'hypertension artérielle et les dyslipémies. Une étude récente a aussi rapporté l'association des intolérances cutanées graves à l'allopurinol avec les comorbidités cardiovasculaires [29]. Le bon sens suggère que l'existence d'une allergie préalable à l'allopurinol puisse être un facteur de risque de réaction grave, Cet élément, qui n'a été que peu étudié, était associé à ces réactions dans deux travaux [28,33], comme dans notre étude, mais pas dans un autre [30].

L'importance de la dose d'allopurinol est diversement appréciée dans la littérature. Certaines études [3, 7, 31, 32], mais non toutes [28] ont noté que le risque de réaction grave augmentait 
avec la posologie d'allopurinol, en particulier chez les insuffisants rénaux [30-32]. Nous n'avons pas trouvé, dans notre étude, d'association avec des posologies plus élevées d'allopurinol. Une étude cas-contrôles rétrospective [34] a conclu que la dose initiale d'allopurinol, et non la dose de maintien thérapeutique, était un facteur de risque de toxicité grave de l'allopurinol et a conduit à la recommandation, reprise par l'ACR [35] et l'EULAR [36], d'augmenter progressivement les doses d'allopurinol au début du traitement, jusqu'à obtention d'une uricémie satisfaisante. L'absence d'augmentation progressive de l'allopurinol parait, dans notre étude, associée ( $\mathrm{p}<0,0001)$ à la survenue d'intolérances graves, mais le petit nombre de patients du groupe contrôle dont l'allopurinol a été augmenté progressivement, en regard l'extrême rareté des réactions graves ne permet pas d'affirmer de façon certaine un effet préventif de l'augmentation progressive de la posologie.

Nous avons trouvé une association significative $(\mathrm{p}<0,01)$ de l'insuffisance rénale avec la survenue de réactions cutanées graves, même si nos résultats nous semblent fragiles du fait de données manquantes chez les patients qui ont développé une toxidermie grave et de la faiblesse globale des effectifs. Le risque lié à l'insuffisance rénale est actuellement un sujet de controverse entre américains et européens [37, 38]. C'est un facteur de risque classique des événements graves [31,32], rapporté à l'augmentation des taux sériques d'oxypurinol chez l'insuffisant rénal, qui a conduit les principales agences d'enregistrement à limiter la posologie d'allopurinol en fonction de la clairance de la créatinine, en suivant les recommandations, déjà anciennes, de Hande et al [31]. Cette limitation empêche de diminuer l'uricémie de beaucoup de goutteux insuffisants rénaux jusqu'à la cible préconisée [39]. Elle est actuellement remise en question en Nouvelle Zélande et aux Etats Unis et n'a pas été prise en compte dans les recommandations thérapeutiques de l'ACR [35]. Le comité en charge des recommandations américaines a indiqué que l'allopurinol pouvait être augmenté de façon très progressive au-delà des doses permises par la règle de Hande [31] jusqu'à obtenir une uricémie satisfaisante. Celui de l'EULAR, conscient des incertitudes persistantes du fait (i) de l'absence d'études prouvant la validité de la prévention par augmentation progressive de la posologie sur des populations suffisamment nombreuses pour prendre en compte la rareté des événements, (ii) de la démonstration récente du rôle aggravant de l'insuffisance rénale sur la mortalité des accidents cutanés [30] et (iii) de l'existence d'alternatives thérapeutiques, a maintenu la limitation de posologie en fonction de la clairance de la créatinine. Nos résultats, du fait leurs importantes limitations et du faible effectif étudié ne nous semblent pas permettre pas de trancher ce débat.

En ce qui concerne les éruptions bénignes connues pour compliquer 2 à $4 \%$ des traitements par l'allopurinol [13, 40, 41], nous n'avons pas trouvé d'association avec 1' HLA-B*5801. Les résultats concernent ici 53 patients. Ce nombre peut être considéré comme encore faible et doit être augmenté, mais il est largement supérieur à ceux des 3 études publiées avant la nôtre, dont l'une, australienne et ayant inclus 12 éruptions maculo-papuleuses, n' a pas trouvé d'association [42], et dont deux, effectuées à Shanghai (22 éruptions bénignes étudiées) [20] et en Thaïlande (6 éruptions) [19] rapportent une association. Si la littérature est riche sur les facteurs de risque 
non HLA des accidents cutanés graves à l'allopurinol, nous n'y avons pas trouvé d'étude concernant les toxidermies bénignes. Notre étude suggère, pour la première fois, que l'absence d'augmentation progressive de la posologie accroit le risque d'éruption bénigne à l'allopurinol. En revanche, la posologie du médicament au moment de l'éruption n'était pas plus élevée et était même significativement plus basse chez les patients qui développèrent une réaction bénigne, que chez ceux dont la tolérance était bonne.

\section{Conclusions}

Ces résultats préliminaires suggèrent que la présence de l' HLA-B*5801est très fortement associée aux syndromes de Lyell et de Stevens-Johnson induits par l' allopurinol, mais pas aux réactions cutanées bénignes à ce médicament dans la population vietnamienne. Ces résultats, s'ils sont confirmés par la poursuite de notre étude, plaideront pour la réalisation d'un génotypage HLA-B*5801chez les goutteux vietnamiens candidats à un traitement par l'allopurinol, non seulement au Vietnam mais aussi dans la communauté d'origine vietnamienne en France.

Remerciements : L'étude a été financée par l'association ART Viggo (hôpital Lariboisière, Paris, France)

\section{Références}

[1] Stamp LK, Day RO, Yun J. Allopurinol hypersensitivity: investigating the cause and minimizing the risk. Nat Rev Rheumatol 2016;12:235-42.

[2] Roujeau JC. Clinical heterogeneity of drug hypersensitivity. Toxicology 2005;209(2):123-9.

[3] Lonjou C, Borot N, Sekula P, Ledger N, Thomas L, Halevy S, et al. A European study of HLA-B in Stevens-Johnson syndrome and toxic epidermal necrolysis related to five high-risk drugs. Pharmacogenet Genomics 2008;18:99-107.

[4] Miliszewski MA, Kirchhof MG, Sikora S, Papp A, Dutz JP. Stevens-Johnson Syndrome and Toxic Epidermal Necrolysis: An Analysis of Triggers and Implications for Improving Prevention. Am J Med 2016;129:1221-1225.

[5] Diphoorn J, Cazzaniga S, Gamba C, Schroeder J, Citterio A, Rivolta AL, et al. Incidence, causative factors and mortality rates of Stevens-Johnson syndrome (SJS) and toxic epidermal necrolysis (TEN) in northern Italy: data from the REACT registry. Pharmacoepidemiol Drug Saf 2016;25:196-203.

[6] Kardaun SH, Sekula P, Valeyrie-Allanore L, Liss Y, Chu CY, Creamer D, et al. Drug reaction with eosinophilia and systemic symptoms (DRESS): an original multisystem adverse drug reaction. Results from the prospective RegiSCAR study. Br J Dermatol 2013;169:1071-80.

[7] Kim SC, Newcomb C, Margolis D, Roy J, Hennessy S. Severe cutaneous reactions requiring hospitalization in allopurinol initiators: a population-based cohort study. Arthritis Care Res (Hoboken) 2013;65:578-84.

[8] Hsu DY, Brieva J, Silverberg NB, Silverberg JI. Morbidity and Mortality of Stevens-Johnson Syndrome and Toxic Epidermal Necrolysis in United States Adults. J Invest Dermatol 2016;136:1387-1397.

[9] Lu N, Rai SK, Terkeltaub R, Kim SC, Menendez ME, Choi HK. Racial disparities in the risk of Stevens-Johnson Syndrome and toxic epidermal necrolysis as urate-lowering drug adverse events in the United States. Semin Arthritis Rheum 2016;46:253-8. 
[10] Hung SI, Chung WH, Liou LB, Chu CC, Lin M, Huang HP, et al. HLA-B*5801 allele as a genetic marker for severe cutaneous adverse reactions caused by allopurinol. Proc Natl Acad Sci U S A 2005; 102:4134-9.

[11] Yun J, Marcaida MJ, Eriksson KK, Jamin H, Fontana S, Pichler WJ, et al. Oxypurinol directly and immediately activates the drug-specific $\mathrm{T}$ cells via the preferential use of HLA-B*58:01. J Immunol 2014;192:2984-93.

[12] Chung WH, Pan RY, Chu MT, Chin SW, Huang YL, Wang WC, et al. Oxypurinol-Specific T Cells Possess Preferential TCR Clonotypes and Express Granulysin in Allopurinol-Induced Severe Cutaneous Adverse Reactions. J Invest Dermatol 2015;135:2237-48.

[13] Ko TM, Tsai CY, Chen SY, Chen KS, Yu KH, Chu CS, et al. Use of HLA-B*58:01 genotyping to prevent allopurinol induced severe cutaneous adverse reactions in Taiwan: national prospective cohort study. BMJ 2015;351:h4848.

[14] Hershfield MS, Callaghan JT, Tassaneeyakul W, Mushiroda T, Thorn CF, Klein TE, et al. Clinical Pharmacogenetics Implementation Consortium guidelines for human leukocyte antigenB genotype and allopurinol dosing. Clin Pharmacol Ther 2013;93:153-8.

[15] Jarjour S, Barrette M, Normand V, Rouleau JL, Dube MP, de Denus S. Genetic markers associated with cutaneous adverse drug reactions to allopurinol: a systematic review. Pharmacogenomics 2015; 16:755-67.

[16] Bardin T. NDQ, Tran Minh K., Le Hieu N., Do Duc M., Richette P., Resche-Rigon M. Urate Lowering to ACR-Recommended Targets Allows Significant Improvement of Severe Gout: A Monocentric Prospective Trial in Vietnam, Using a Systematic Treatment Protocol [abstract] Arthritis Rheumatol. http://acrabstracts.org/abstract/urate-lowering-to-acr-recommended-targetsallows-significant-improvement-of-severe-gout-a-monocentric-prospective-trial-in-vietnamusing-a-systematic-treatment-protocol/. 2017;69(suppl 10).

[17] Kardaun SH, Sidoroff A, Valeyrie-Allanore L, Halevy S, Davidovici BB, Mockenhaupt M, et al. Variability in the clinical pattern of cutaneous side-effects of drugs with systemic symptoms: does a DRESS syndrome really exist? Br J Dermatol 2007;156:609-11.

[18] Goncalo M, Coutinho I, Teixeira V, Gameiro AR, Brites MM, Nunes R, et al. HLA-B*58:01 is a risk factor for allopurinol-induced DRESS and Stevens-Johnson syndrome/toxic epidermal necrolysis in a Portuguese population. Br J Dermatol 2013;169:660-5.

[19] Sukasem C, Jantararoungtong T, Kuntawong P, Puangpetch A, Koomdee N, Satapornpong P, et al. HLA-B (*) 58:01 for Allopurinol-Induced Cutaneous Adverse Drug Reactions: Implication for Clinical Interpretation in Thailand. Front Pharmacol 2016;7:186.

[20] Cao ZH, Wei ZY, Zhu QY, Zhang JY, Yang L, Qin SY, et al. HLA-B*58:01 allele is associated with augmented risk for both mild and severe cutaneous adverse reactions induced by allopurinol in Han Chinese. Pharmacogenomics 2012;13:1193-201.

[21] Chiu ML, Hu M, Ng MH, Yeung CK, Chan JC, Chang MM, et al. Association between HLA$\mathrm{B} * 58: 01$ allele and severe cutaneous adverse reactions with allopurinol in Han Chinese in Hong Kong. Br J Dermatol 2012;167:44-9.

[22] Jung JW, Song WJ, Kim YS, Joo KW, Lee KW, Kim SH, et al. HLA-B58 can help the clinical decision on starting allopurinol in patients with chronic renal insufficiency. Nephrol Dial Transplant 2011;26:3567-72.

[23] Do TQN, Tran, T.H.A., Vu, T.K.L., Tran, Q.B., Nguyen, T.H.N., Chu, C.H., Nguyen, V.D., Tran, Q.B., Nguyen, T.T.H., Le, T.M.H. Association between HLA*B5801 and allopurinol-induced severe cutaneous adverse drug reaction in Bachmai hospital. Tap chi $\mathrm{Y}$ hoc du phong 2015;25:396_400.

[24] Kaniwa N, Saito Y, Aihara M, Matsunaga K, Tohkin M, Kurose K, et al. HLA-B locus in Japanese patients with anti-epileptics and allopurinol-related Stevens-Johnson syndrome and toxic epidermal necrolysis. Pharmacogenomics 2008;9:1617-22. 
[25] Lonjou C, Borot N, Sekula P, Ledger N, Thomas L, Halevy S, et al. A European study of HLA-B in Stevens-Johnson syndrome and toxic epidermal necrolysis related to five high-risk drugs. Pharmacogenet Genomics 2008;18:99-107.

[26] Hoa BK, Hang NT, Kashiwase K, Ohashi J, Lien LT, Horie T, et al. HLA-A, -B, -C, -DRB1 and DQB1 alleles and haplotypes in the Kinh population in Vietnam. Tissue Antigens 2008;71:12734.

[27] Nga D. T. Q. ATTH, Lien V.T.K., Binh T.Q., Nhung N. T. H., Hieu C.C., . association between HLAB*5801 and allopurinol-induced severe cutaneous reactions in Bachmai hospital. Tap chi Y hoc du phong 2015;25:396-400.

[28] Ramasamy SN, Korb-Wells CS, Kannangara DR, Smith MW, Wang N, Roberts DM, et al. Allopurinol hypersensitivity: a systematic review of all published cases, 1950-2012. Drug Saf 2013;36:953-80.

[29] Yang CY, Chen CH, Deng ST, Huang CS, Lin YJ, Chen YJ, et al. Allopurinol Use and Risk of Fatal Hypersensitivity Reactions: A Nationwide Population-Based Study in Taiwan. JAMA Intern Med 2015;175(9):1550-7.

[30] Chung WH, Chang WC, Stocker SL, Juo CG, Graham GG, Lee MH, et al. Insights into the poor prognosis of allopurinol-induced severe cutaneous adverse reactions: the impact of renal insufficiency, high plasma levels of oxypurinol and granulysin. Ann Rheum Dis 2015;74:215764.

[31] Hande KR, Noone RM, Stone WJ. Severe allopurinol toxicity. Description and guidelines for prevention in patients with renal insufficiency. Am J Med 1984;76(1):47-56.

[32] Lupton GP, Odom RB. The allopurinol hypersensitivity syndrome. J Am Acad Dermatol 1979;1:365-74.

[33] Chaby G, Valeyrie-Allanore L, Duong TA, Lebrun-Vignes B, Milpied B, Sassolas B, et al. Severe cutaneous adverse reactions due to inappropriate medication use. Br J Dermatol 2018.

[34] Stamp LK, Taylor WJ, Jones PB, Dockerty JL, Drake J, Frampton C, et al. Starting dose is a risk factor for allopurinol hypersensitivity syndrome: a proposed safe starting dose of allopurinol. Arthritis Rheum 2012;64:2529-36.

[35] Khanna D, Fitzgerald JD, Khanna PP, Bae S, Singh MK, Neogi T, et al. 2012 American College of Rheumatology guidelines for management of gout. Part 1: systematic nonpharmacologic and pharmacologic therapeutic approaches to hyperuricemia. Arthritis Care Res (Hoboken) 2012;64:1431-46.

[36] Richette P, Doherty M, Pascual E, Barskova V, Becce F, Castaneda-Sanabria J, et al. 2016 updated EULAR evidence-based recommendations for the management of gout. Ann Rheum Dis 2017;76:29-42.

[37] Neogi T, Dalbeth N, Stamp L, Castelar G, Fitzgerald J, Gaffo A, et al. Renal dosing of allopurinol results in suboptimal gout care. Ann Rheum Dis 2017;76:e1.

[38] Richette P, Doherty M, Pascual E, Bardin T. Response: Renal dosing of allopurinol results in suboptimal gout care by T Neogi et al. Ann Rheum Dis 2017;76:e2.

[39] Stamp LK, Merriman TR, Barclay ML, Singh JA, Roberts RL, Wright DF, et al. Impaired response or insufficient dosage? Examining the potential causes of "inadequate response" to allopurinol in the treatment of gout. Semin Arthritis Rheum 2014;44:170-4.

[40] McInnes GT, Lawson DH, Jick H. Acute adverse reactions attributed to allopurinol in hospitalised patients. Ann Rheum Dis 1981;40:245-9.

[41] Hoigne R, Sonntag MR, Zoppi M, Hess T, Maibach R, Fritschy D. Occurrence of exanthems in relation to aminopenicillin preparations and allopurinol. N Engl J Med 1987;316:1217.

[42] Lee MH, Stocker SL, Anderson J, Phillips EJ, Nolan D, Williams KM, et al. Initiating allopurinol therapy: do we need to know the patient's human leucocyte antigen status? Intern Med J 2012;42:411-6. 
Tableau 1 : Caractéristiques des patients avec allergies sévères ou bénignes à l'allopurinol et de ceux ayant bien toléré le médicament. Les variables qualitatives sont décrites par leurs effectifs et pourcentages $\mathrm{N}(\%)$ et les variables quantitatives par leurs moyennes et écart-types (ET).

\begin{tabular}{|c|c|c|c|c|c|c|}
\hline & $\begin{array}{l}\text { Lyell/ } \\
\text { Stevens- } \\
\text { Jonhson } \\
(\mathrm{n}=10)\end{array}$ & $\begin{array}{l}\text { Intolérances } \\
\text { bénignes } \\
(\mathrm{n}=54)\end{array}$ & $\begin{array}{l}\text { Bonne } \\
\text { tolérance } \\
(\mathrm{n}=112)\end{array}$ & $\begin{array}{l}\text { Valeur de } \\
\mathrm{p} \\
\text { (test } \\
\text { global) }\end{array}$ & $\begin{array}{l}\text { Valeur de p } \\
\text { Lyell/Steve } \\
\text { ns-Johnson } \\
\text { vs bonne } \\
\text { tolérance }\end{array}$ & $\begin{array}{l}\text { Valeur de p } \\
\text { Intolérances } \\
\text { bénignes vs } \\
\text { bonne } \\
\text { tolérance }\end{array}$ \\
\hline $\begin{array}{l}\text { Absence de } \\
\text { goutte }\end{array}$ & $\begin{array}{c}5 \\
(50 \%)\end{array}$ & $\begin{array}{c}53 \\
(98 \%)\end{array}$ & $\begin{array}{c}112 \\
(100 \%)\end{array}$ & $\mathrm{P}<0,0001$ & $\mathrm{P}<0,0001$ & $\mathrm{P}=0,28$ \\
\hline $\begin{array}{l}\text { Femmes } \\
\mathrm{N}(\%)\end{array}$ & $\begin{array}{c}2 \\
(20 \%)\end{array}$ & 0 & 0 & $P=0,003$ & $P=0,006$ & $\mathrm{P}=1$ \\
\hline $\begin{array}{l}\text { Age } \\
\text { Moy (ET) }\end{array}$ & $\begin{array}{c}55 \\
(12)\end{array}$ & $\begin{array}{c}47 \\
(12)\end{array}$ & $\begin{array}{c}46 \\
(12)\end{array}$ & $P=0,012$ & $P=0,04$ & $\mathrm{P}=0,73$ \\
\hline $\begin{array}{l}\text { Antécédent } \\
\text { d'allergie à } \\
\text { l'allopurinol }\end{array}$ & $\begin{array}{c}1 / 7 \\
(14 \%)\end{array}$ & $\begin{array}{l}1 / 52 \\
(2 \%)\end{array}$ & 0 & $\mathrm{P}<0,0001$ & $\mathrm{P}=0,006$ & $\mathrm{P}=0,32$ \\
\hline $\begin{array}{l}\text { Prise de } \\
\text { diurétique }\end{array}$ & $\begin{array}{c}2 / 9 \\
(22 \%)\end{array}$ & $\begin{array}{l}1 / 52 \\
(2 \%)\end{array}$ & 0 & $P=0,002$ & $P=0.005$ & $\mathrm{P}=0,32$ \\
\hline $\begin{array}{l}\text { Dose } \\
\text { allopurinol } \\
\text { moy (ET) }\end{array}$ & $\begin{array}{c}345 \mathrm{mg} / \mathrm{j} \\
(142)\end{array}$ & $\begin{array}{c}259 \mathrm{mg} / \mathrm{j} \\
(119)\end{array}$ & $\begin{array}{c}356 \mathrm{mg} / \mathrm{j} \\
(114)\end{array}$ & $P<0,0001$ & $\mathrm{P}<0,0001$ & $\mathrm{P}=0.51$ \\
\hline $\begin{array}{l}\text { Doses } \\
\text { progressives }\end{array}$ & 0 & $\begin{array}{c}49 \\
(91 \%)\end{array}$ & $\begin{array}{c}112 \\
(100 \%)\end{array}$ & $P<0,0001$ & $\mathrm{P}<0,0001$ & $\mathrm{P}<0,0001$ \\
\hline
\end{tabular}

\begin{tabular}{|l|c|c|c|c|c|c|}
\hline $\begin{array}{l}\text { DFG < 60 } \\
\text { ml/min }\end{array}$ & $\begin{array}{c}2 / 6 \\
(33 \%)\end{array}$ & $\begin{array}{c}1 / 51 \\
(0,2 \%)\end{array}$ & $\begin{array}{c}1 / 88 \\
(0,01 \%)\end{array}$ & $\mathrm{P}=0,008$ & $\mathrm{P}=0,01$ & $\mathrm{P}=1$ \\
\hline $\begin{array}{l}\text { Diabète de } \\
\text { type 2 }\end{array}$ & $1 / 6$ & $\begin{array}{c}2 / 54 \\
(3,7 \%)\end{array}$ & $\begin{array}{c}2 / 112 \\
(1,8 \%)\end{array}$ & $\mathrm{P}=0,13$ & & \\
\hline HTA & $3 / 6$ & $\begin{array}{c}14 / 54 \\
(50 \%)\end{array}$ & $\begin{array}{c}13 / 112 \\
(11,6 \%)\end{array}$ & $\mathrm{P}=0,006$ & $\mathrm{P}=0,03$ & $\mathrm{P}=0,03$ \\
\hline $\begin{array}{l}\text { Insuffisance } \\
\text { coronarienne }\end{array}$ & $0 / 6$ & $\begin{array}{c}1 / 54 \\
(1,8 \%)\end{array}$ & $\begin{array}{c}1 / 112 \\
(0,9 \%)\end{array}$ & $\mathrm{P}=0,58$ & & \\
\hline dyslipidémie & $3 / 6$ & $\begin{array}{c}36 / 54 \\
(66.7 \%)\end{array}$ & $\begin{array}{c}26 / 112 \\
(23 \%)\end{array}$ & $\mathrm{P}<0,0001$ & $\mathrm{P}=0,015$ & $\mathrm{P}<0,0001$ \\
\hline $\begin{array}{l}\text { HLA } \\
\mathrm{B} * 5801\end{array}$ & $\begin{array}{c}3 \\
(100 \%)\end{array}$ & $\begin{array}{c}(5,6 \%) \\
(5,3 \%)\end{array}$ & $\mathrm{P}<0,0001$ & $\mathrm{P}<0,0001$ & $\mathrm{P}=1$ \\
\hline
\end{tabular}


Figure 1 : Syndrome de Lyell/Stevens Johnson chez un des patients inclus dans l'étude

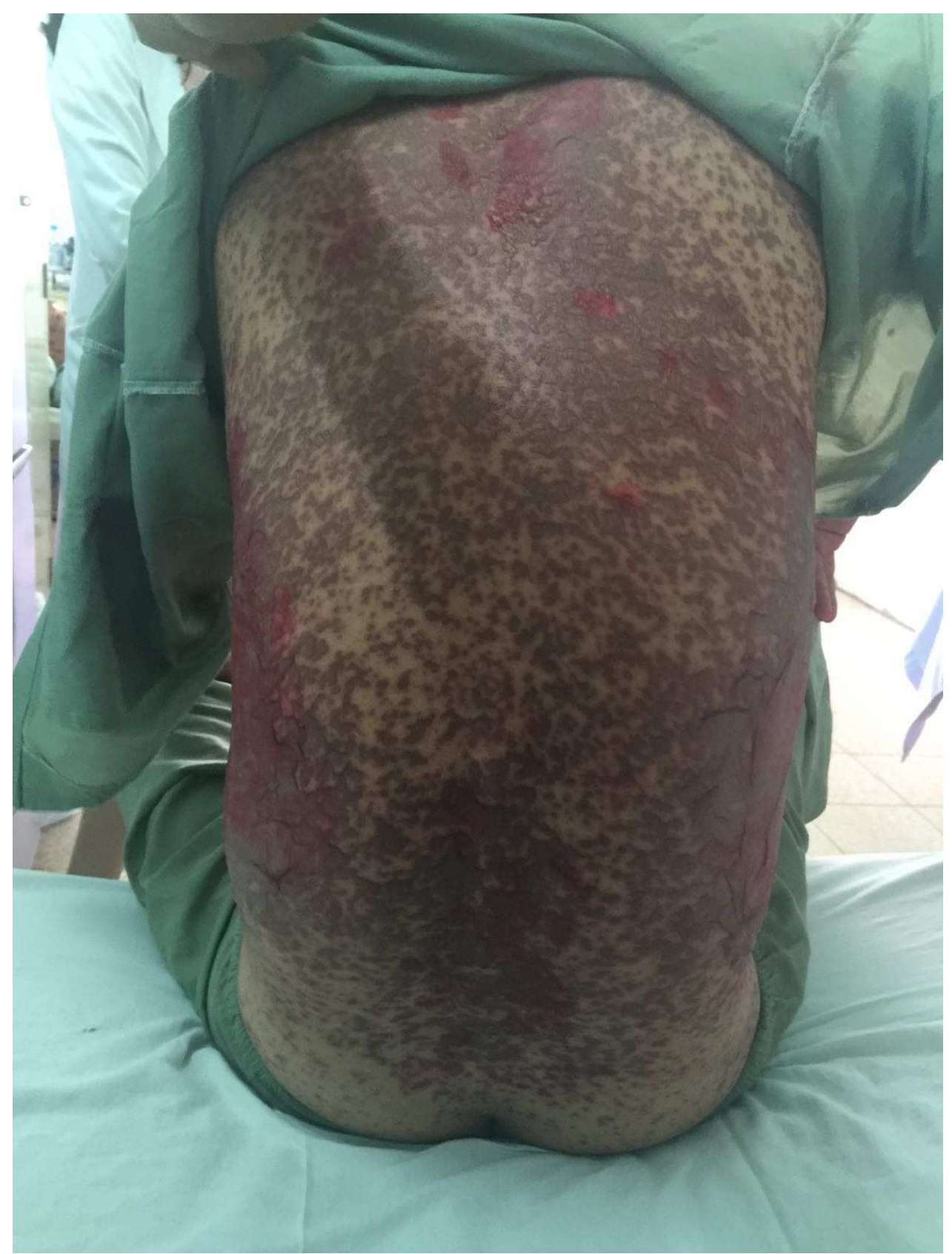

